\title{
A Study of Quasi-Monochromatic X-Ray Sources for Breast Cancer Early Detection
}

\author{
Xin Qian'1, Lindsay Puckett' ${ }^{1}$ Zhijun Cai ${ }^{2}$ \\ ${ }^{1}$ Department of Radiation Medicine, Northwell Health, Great Neck, NY, USA \\ ${ }^{2}$ Department of Radiology, University of Iowa Hospitals and Clinics, Iowa City, IA, USA \\ Email: xqian@northwell.edu
}

How to cite this paper: Qian, X., Puckett, L. and Cai, Z.J. (2018) A Study of Quasi-Monochromatic X-Ray Sources for Breast Cancer Early Detection. International Journal of Medical Physics, Clinical Engineering and Radiation Oncology, 7, 184-192.

https://doi.org/10.4236/ijmpcero.2018.72016

Received: April 8, 2018

Accepted: May 19, 2018

Published: May 22, 2018

Copyright $\odot 2018$ by authors and Scientific Research Publishing Inc. This work is licensed under the Creative Commons Attribution International License (CC BY 4.0).

http://creativecommons.org/licenses/by/4.0/

\begin{abstract}
Objectives: Early detection and treatment provide the opportunity to decrease the mortality rate from breast cancer. Quasi-monochromatic technique can enhance low contrast lesion detection by eliminating beam-hardening artifacts. We hypothesized that this technique would be feasible and can be used to assist in breast cancer early detection. Methods: The performance of stationary Digital Breast Tomosynthesis with quasi-monochromatic X-ray sources was evaluated using both simulated and physical phantoms. Normalized spectra in the fraction of total photons vs. photon energy were generated. Results: As expected, the peaked energies from sources, from smallest to largest, are $\mathrm{Mo} / \mathrm{Mo}, \mathrm{W} / \mathrm{Ag}-1000$, and W/Ce-10, W/Ce-100. For contrast vs. noise standard deviation on the simulated CIRS phantom, W/Ce-100 and W/Ce-10 have similar performance on both low and high contrast objects. For low contrast object, $\mathrm{W} / \mathrm{Ce}-100$ is barely noticeably better than W/Ce-10, and they are better than both W/Ag-1000 and Mo/Mo. For high contrast objects W/Ce-10 is slightly better than W/Ce-100. The spectra of the implemented W/Ce-10 X-ray source were measured, which matched the simulation well. The contrast noise ratios of reconstructed objects in American College of Radiology mammographic phantom with and without using W/Ce-10 combination are 7.1 and 5.4, respectively. Conclusions: The combination of stationary digital breast tomosynthesis and quasi-monochromatic technique can compensate the loss of X-ray flux due to heavy K-edge filtering. This technique can enable the stationary DBT scanners to operate at acceptable scanning times with better low contrast lesion detectability. Advances in Knowledge: The stationary digital breast tomosynthesis can provide high quality images within short scanning time by using X-ray source array, which makes quasi-monochromatic technique feasible.
\end{abstract}

\section{Keywords}

Breast Cancer, Quasi-Monochromatic, Early Detection, Digital Breast 
Tomosynthesis

\section{Introduction}

Digital breast tomosynthesis (DBT) is a three-dimensional (3D) imaging technique that provides the reconstruction of an arbitrary set of planes in the breast from limited-angle series of projection images as the X-ray tube moves. Compared with traditional two-dimensional (2D) mammography, 3D tomosynthesis imaging methods have the potential to improve conspicuity of structures by removing the visual clutter associated with overlying anatomy [1] [2] [3]. The DBT reconstruction methods make it feasible to detect lesions at smaller size and earlier stage, thereby potentially helping to reduce the number of women who die from breast cancer each year [4]-[11].

To further improve the DBT overall image quality, we have studied quasi-monochromatic imaging technique. The major advantages of this method include: 1) Eliminating beam-hardening artifacts and therefore improving the ability to detect low contrast lesions; 2) Allowing more accurate quantification of absorption coefficients; 3) The beam energy can be used for K-edge imaging and thus improves image contrast. While the above-mentioned advantages associated with quasi-monochromatic X-ray imaging are obvious, they have not been realized for clinical uses. The main reason for this is the lack of monochromatic or quasi-monochromatic X-ray sources that can produce sufficient flux for practical applications. Most of the studies reported so far relied on intense $\mathrm{X}$-ray radiation generated by synchrotron X-ray facilities [1] [12] [13] [14] [15]. Unfortunately, synchrotron generated X-ray sources are both limited in availability and associated with high costs, making it difficult to be widely utilized for clinical and research applications.

The feasibility of generating quasi-monochromatic X-ray radiation by K-edge filtering polychromatic beam using selected high $\mathrm{Z}$ (atomic number) materials has recently been studied by simulation [16] and by experiment [17]. One major advantage of this method for cone beam imaging is that the filtered X-ray maintains the cone-beam geometry. However, the loss of $99.5 \%$ of photon flux (for $200^{\text {th }}$ value layer) still makes the process impractical for clinical use due to the long exposure time it would demand for even when utilizing a high power rotating anode $\mathrm{X}$-ray source.

The combination of stationary DBT design [11] and quasi-monochromatic imaging technique could potentially compensate the loss of X-ray flux due to the use of heavy K edge filtering. This would enable the stationary DBT scanner to operate at a comparable scanning time as today's C-arm based system, but with a better imaging quality and a reduced imaging dose. In this study, we focused on applying quasi-monochromatic imaging method to improve stationary DBT overall image quality. 


\section{Methods and Materials}

\subsection{Simulation of Quasi-Monochromatic Cone-Beam X-Ray by K-Edge Filtering}

Three possible quasi-monochromatic configurations have been simulated to evaluate the effects of different energy spectra on stationary DBT image quality. In this study, we compared a conventional Mo/Mo (molybdenum anode, molybdenum filter) source with the following K-edge-filtered approaches for 32 $\mathrm{keV}:$ 1) tungsten source with cerium filter at the $100^{\text {th }} \mathrm{VL}$, (W/Ce-100); 2) tungsten source with cerium filter at the $\left.10^{\text {th }} \mathrm{VL},(\mathrm{W} / \mathrm{Ce}-10) ; 3\right)$ tungsten source with silver filter at the $1000^{\text {th }} \mathrm{VL},(\mathrm{W} / \mathrm{Ag}-1000)$. The details of these configurations are given in Table 1.

\subsection{Simulation of CIRS Phantom and Reconstructed Data Using Tomosynthesis System}

Polychromatic X-ray tomosynthesis data for a configuration like the one currently being implemented for the stationary DBT system were simulated using the polychromatic ray-tracing method [15]. The simulated subject was a computer construct of the CIRS phantom, which includes both low-contrast masses and high-contrast calcifications embedded in a material resembling compressed breast, as shown in Figure 1. The polychromatic simulation accounts for energy dependence of both the spectrum and of the materials' attenuation factors, as drawn from the NIST ESTAR database [17]. For each energy in the spectrum, the beam attenuation is computed from the line length of the beam through the object and the linear attenuation coefficient of its material, determined from the material composition and the mass attenuation coefficients of elemental media in the NIST database. The simulation also has the capability to produce realistic noise models for energy-integrating detectors that follow the compound Poisson model. Simulated projection data were computed for the 15-beam system for the three target/filter combinations as described above. The detector pixel size was $127 \mathrm{um}$, with $500 \times 1100$ pixels required to fully image the phantom at all views. These data were reconstructed using the filtered back projection algorithm.

We focused on the W/Ce-10 case in this study. Cone beam quasi-monochromatic $\mathrm{X}$-ray can be produced by heavy filtering using the stationary X-ray tube [11] and spectra data can be obtained using the XR100T-CdTe digital X-ray detector (AmpTek, Inc.), shown in Figure 2(a) \& Figure 2(b)). The peak voltage of the

Table 1. Configuration and performance data for the X-ray simulations.

\begin{tabular}{ccccc}
\hline Case & $\begin{array}{c}\text { Filter Thickness } \\
(\mathrm{mm})\end{array}$ & $\begin{array}{c}\text { Photon yield at } 750 \mathrm{~mm} \\
\left(\text { Photons } /\left(\mathrm{mAs}^{*} \mathrm{~mm}^{2}\right)\right)\end{array}$ & $\begin{array}{c}\text { Photon yield } \\
\text { ratio }\end{array}$ & $\begin{array}{c}\text { mAs per view } \\
\text { required }\end{array}$ \\
\hline Mo/Mo & 0.60 & 276,793 & 1.00 & 3.20 \\
W/Ce-100 & 0.77 & 24,912 & 0.09 & 35.60 \\
W/Ce-10 & 0.39 & 171,247 & 0.62 & 5.17 \\
W/Ag-1000 & 0.21 & 33,296 & 0.12 & 26.60 \\
\hline
\end{tabular}




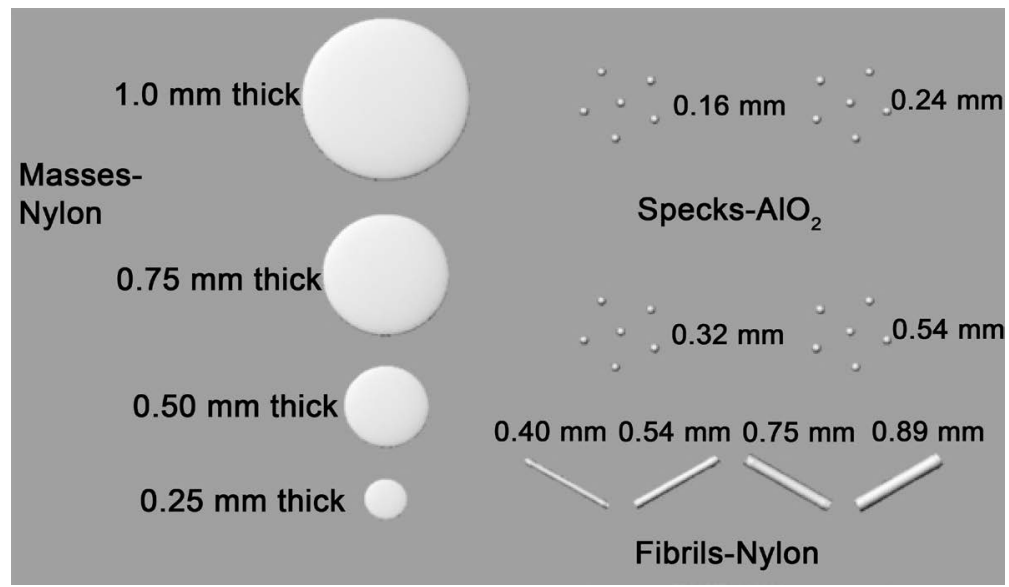

Figure 1. Computer construct of the CIRS digital mammography accreditation phantom.

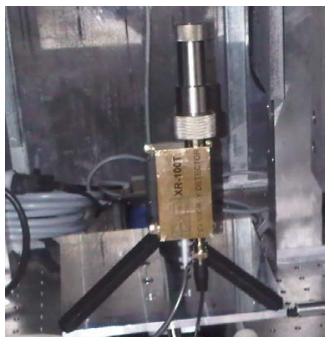

(a)

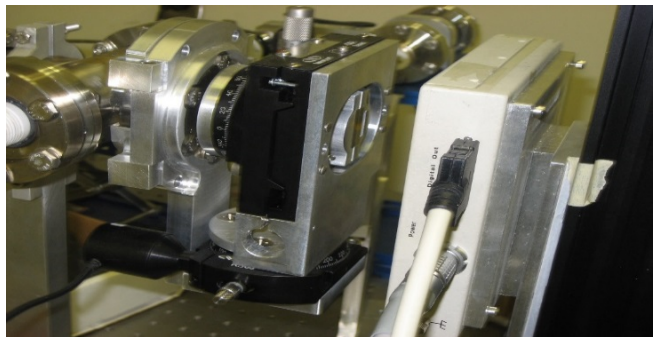

(b)

Figure 2. (a) XR 100T-CdTe X-ray detector; (b) X-ray tube with tungsten target and 0.8 $\mathrm{mm}$ Be window, and filtration holder.

$\mathrm{X}$-ray tube is $80 \mathrm{kVp}$, target is tungsten and tube window is $0.8 \mathrm{~mm} \mathrm{Be}$.

\section{Results}

\subsection{Creation of Quasi-Monochromatic Cone-Beam X-Ray by K-Edge Filtering}

Quantitative results from simulating these spectra using the SPS-78 Spectrum Processor [15] and data from the NIST database [16] are shown in Table 1 along with configurations of source and filters.

Simulations demonstrate that any of these combinations are feasible. While more highly filtered cases will require longer scan times, all can be completed within 15 seconds. The normalized spectra from these cases are shown in Figure 3. While we originally sought to examine cerium filtering only, the silver-filtered case provides a nice compromise between the cerium-filtered spectra and the Mo/Mo case. Figure 4 shows the obtained spectra of combination W/Ce. The background spectra have been subtracted. The experimental result matches well with the simulated one shown in Figure 3.

\subsection{Image Quality of Quasi-Monochromatic Beam Using an ACR Mammographic Phantom}

Noise-free and noisy reconstruction results are shown in Figure 5. In general, 
we found that the contrast of masses was higher for the W/Ce systems while the contrast of the calcifications was higher for the Mo/Mo system. The W/Ce-100 and W/Ce-10 systems provided images with no noticeable difference in visual quality. For each reconstruction image, contrast of both masses and calcifications were computed, as well as the noise standard deviation in uniform image

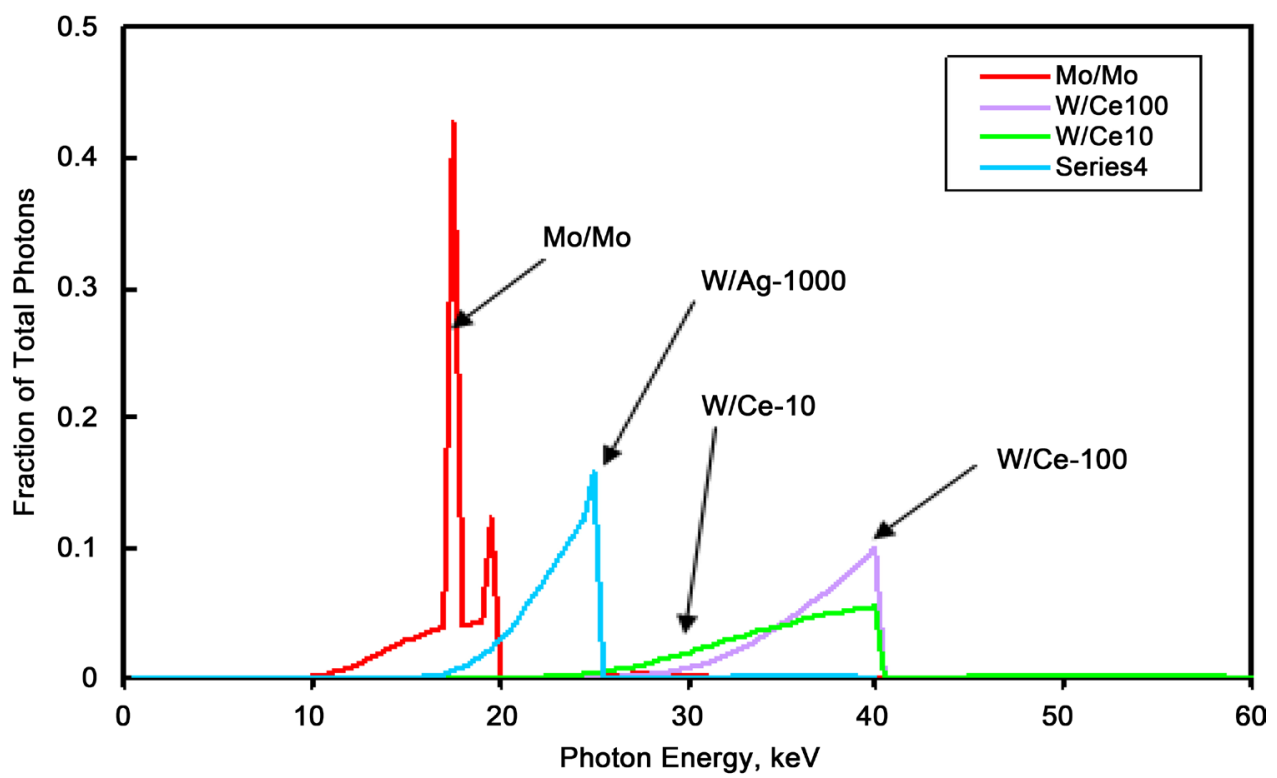

Figure 3. Normalized spectra for each of the four cases studied.

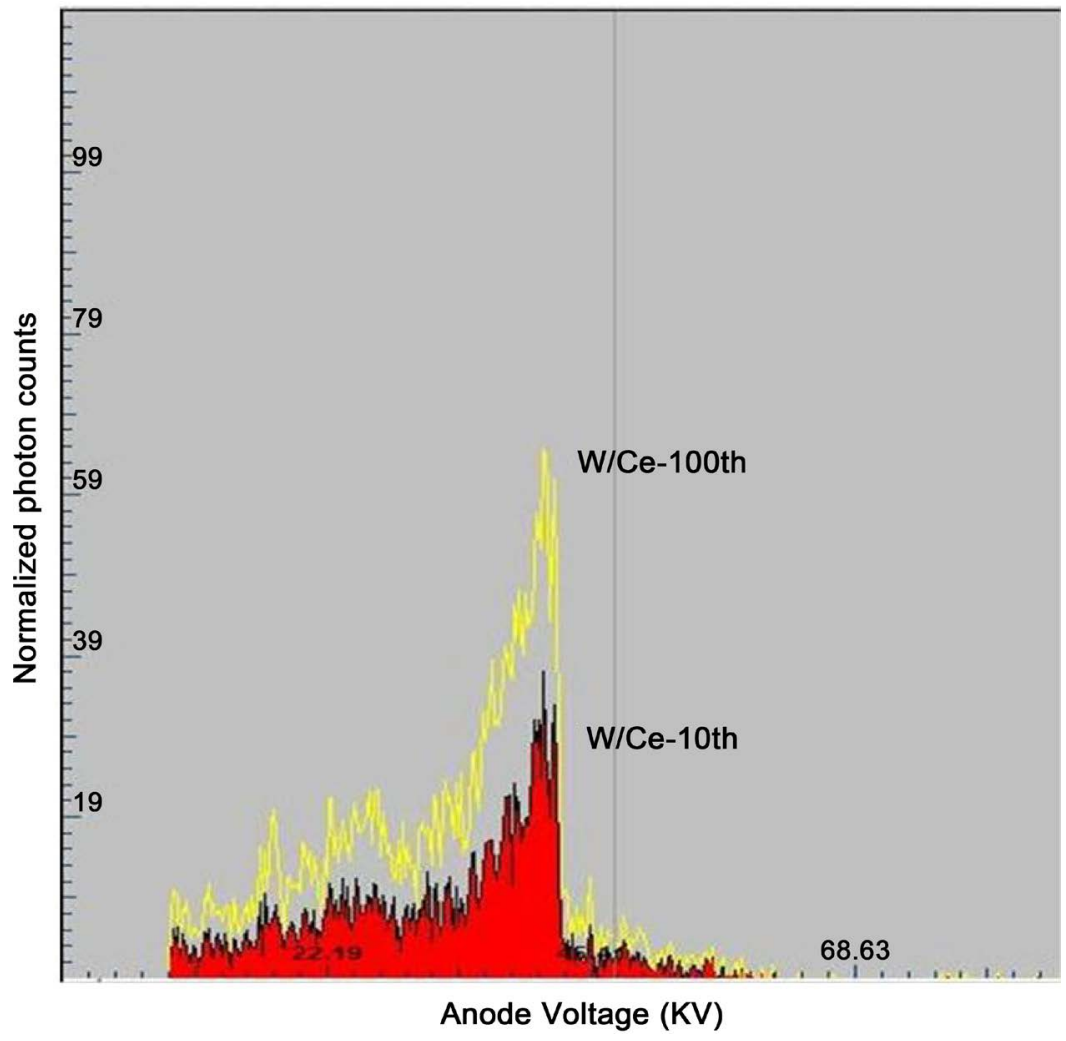

Figure 4. Spectra of $\mathrm{W} / \mathrm{Ce}-100^{\text {th }}$ and $\mathrm{W} / \mathrm{Ce}-10^{\text {th }}$ combinations. 


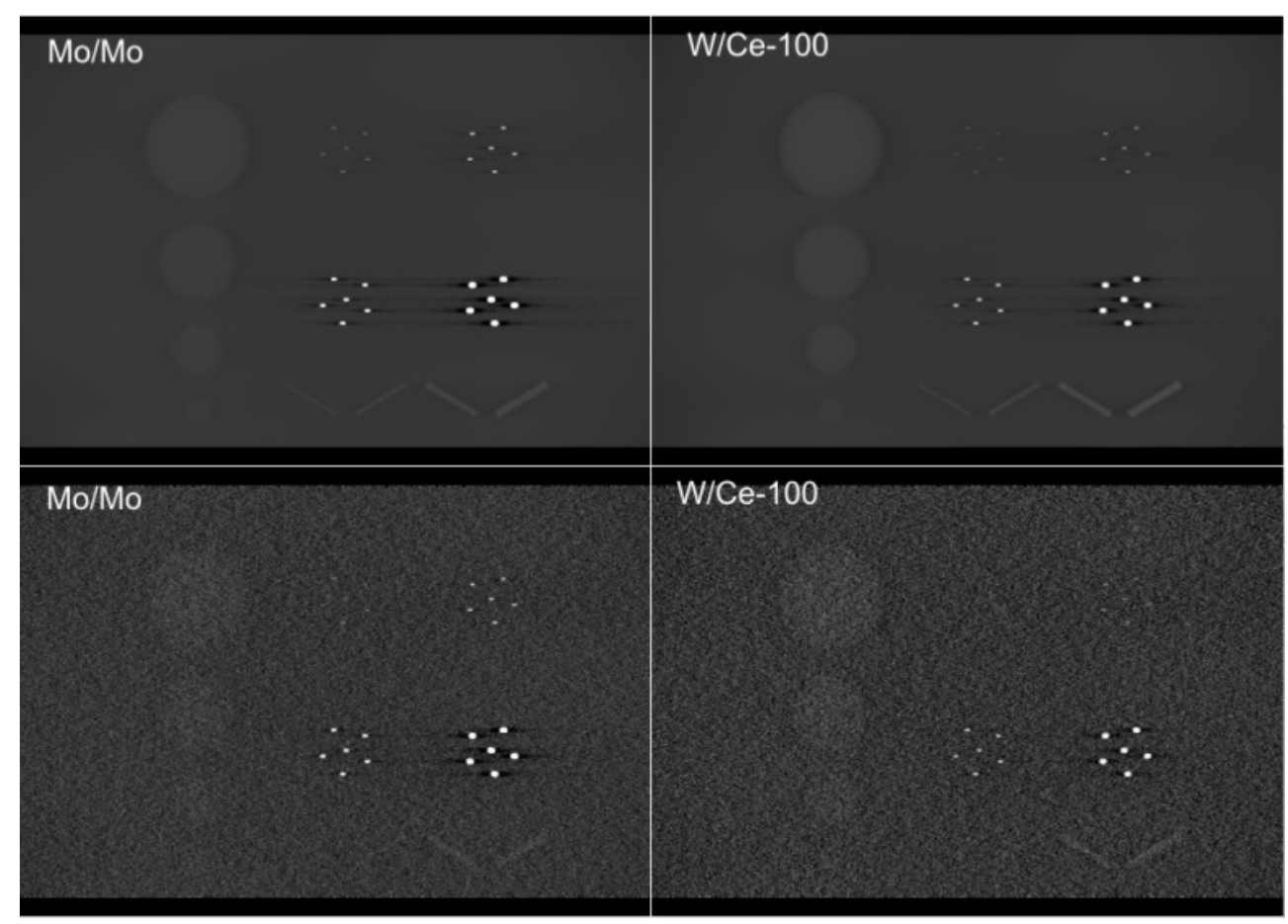

Figure 5. Tomosynthesis reconstructions from simulated data. Top row: Noise-free data; Bottom row: Noisy data.

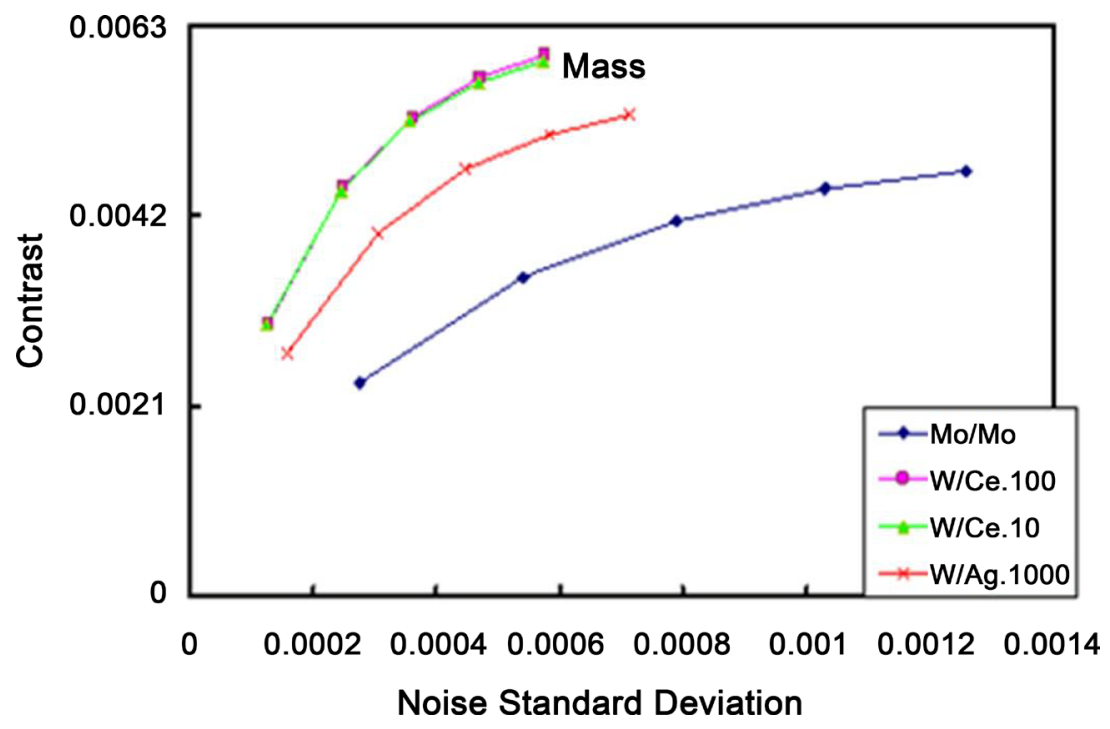

Figure 6. Contrast versus noise performance for a mass in the CIRS phantom construct. Each line shows the results for 2 - 10 iterations of the reconstruction algorithm.

regions. Examples from the data are shown in Figure 6 and Figure 7. Figure 6 presents object contrast versus noise for a low-contrast mass, while Figure 7 does the same for a small calcification $(0.24 \mathrm{~mm})$. These results are typical of masses and calcifications of other sizes. Contrast of masses was about $30 \%$ higher for the W/Ce systems as compared to the Mo/Mo system.

The ACR phantom simulates the X-ray attenuation of a $4.2 \mathrm{~cm}$ slab of compressed human breast composed of 50\% adipose tissue and 50\% glandular tissue. 
Target objects in ACR phantom are six nylon fibrils, five simulated micro-calcification specs, and five masses. All of them are of known size, shape, and density. The layout of target objects in Gammex 156 ACR phantom is shown in Figure 8 (b). The projection images were then reconstructed using the

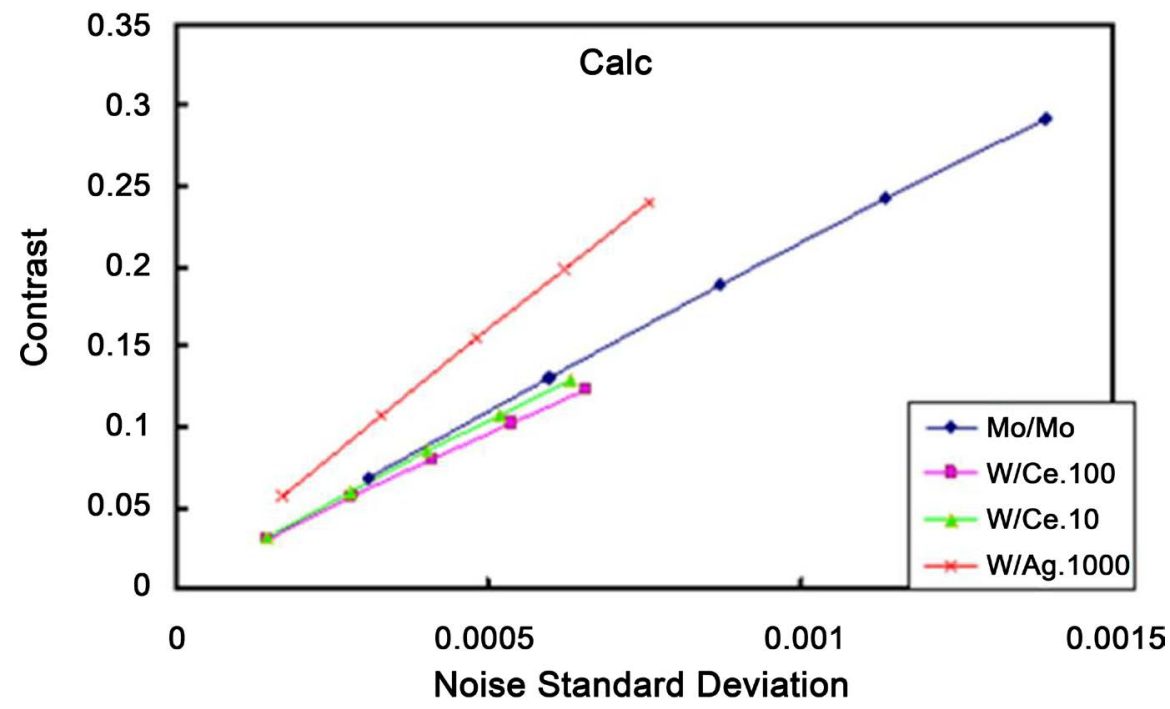

Figure 7. Contrast versus noise performance for a calcification in the CIRS phantom construct. Each line shows the results for 2 - 10 iterations of the reconstruction algorithm.

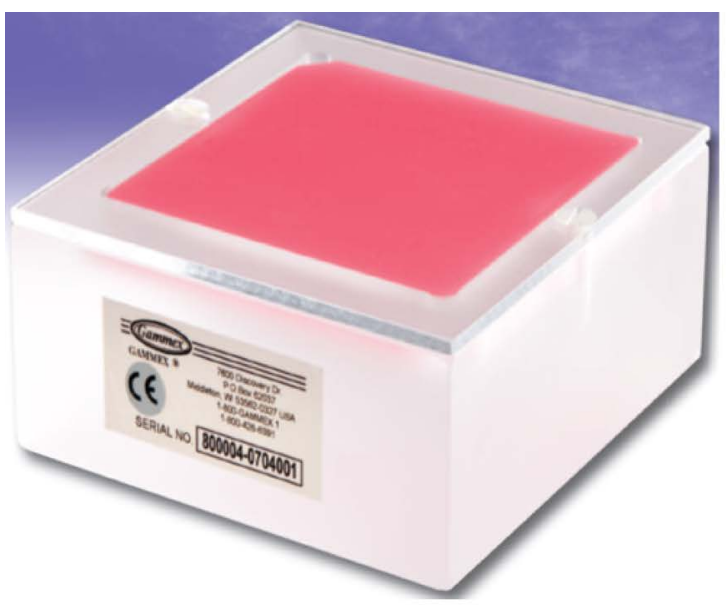

(a)

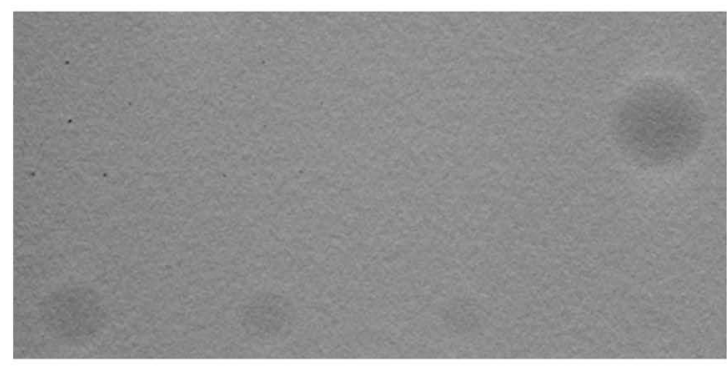

(c)

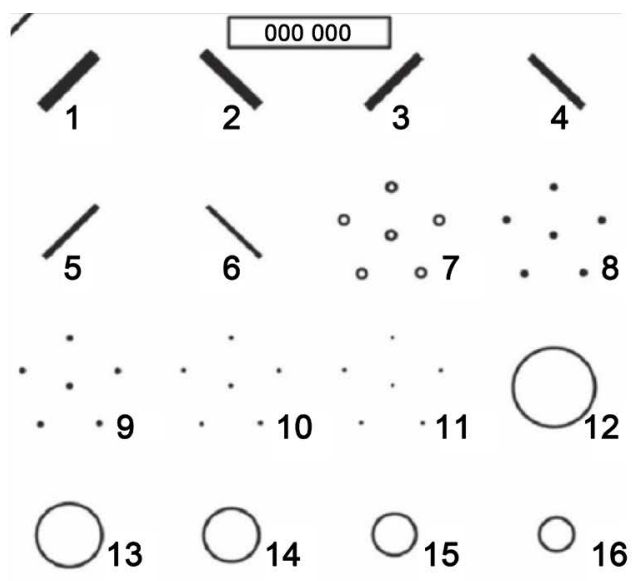

(b)

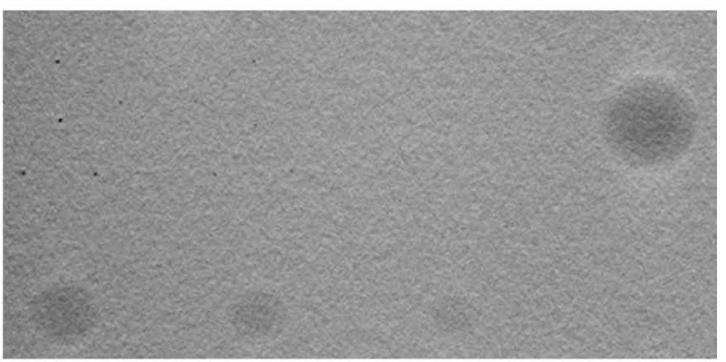

(d)

Figure 8. (a) Gammex 156 ACR phantom; (b) Layout of target objects in Gammex 156 ACR phantom; (c) Without W/Ce10 combination; (d) With W/Ce10 combination. 
back projection (BP) method and the calculated geometry parameters, yielded 50 slices through the phantom. The slice thickness was $1 \mathrm{~mm}$. The images were collected using the following parameters: 15 views over 14 degrees, $28 \mathrm{kVp}$ anode voltage, and a total dose of $100 \mathrm{mAs}$ (6.67 mAs per view).

For comparison, Figure 8(c) and Figure 8(d) show a focused slice of the ACR phantom reconstructed using the data collected from the s-DBT system with the combination $\mathrm{W} / \mathrm{Ce}-100^{\text {th }}$ and without filtration added respectively. The same magnification factor and the window/level are used in displaying the two sets of data. The dashed circles in the images show where the data was taken for the contrast noise ratio (CNR) analysis. CNR was calculated as [lesion - background]/STD. The CNR for Figure 8(c) and Figure 8(d) are 5.4 and 7.1 respectively. Since the exposure was the same for both cases, the exposure efficiency is better by using combination W/Ce-10 than without using filtration.

\section{Discussions and Conclusions}

The simulation results offer two important observations. First, there is little difference in imaging performance between the W/Ce-100 and W/Ce-10 cases. Thus, there may be little to be gained in the increased system power and imaging time required for the W/Ce-100 cases; the W/Ce-10 will perform similarly. Second, the W/Ag-1000 case may be a viable alternative. W/Ag-1000 outperforms the Mo/Mo case by providing better contrast for the same noise level. The comparison between W/Ag-1000 and the W/Ce cases is less clear-cut; W/Ce performs better for masses while W/Ag performs better for calcifications. This is the reason we focused on the W/Ce-10 case in this study. The simulation studies do not account for scatter, for example, which will be a critical factor. Further study of the scatter fraction for each case will need to be completed with Monte Carlo simulation. Also, the cases were not compared for constant dose, but rather for constant photon flux on the subject, so neither dose nor source current is equal. Further study of these factors will be necessary.

In this study, the quasi-monochromatic imaging technique has been tested. Cone beam quasi-monochromatic radiation has been produced by heavy filtering using the stationary X-ray tube with Ce10 filtration. From the experimental data we can observe that the combination W/Ce10 can be used for DBT imaging with reasonable image quality. Contrast noise ratios are improved from 5.4 to 7.1 for low contrast masses.

The combination of the gains from the stationary DBT design and quasi-monochromatic imaging technique can compensate the loss of X-ray flux due to the use of heavy $\mathrm{K}$ edge filtering ( $10^{\text {th }}$ value layer) which enables the stationary DBT scanner to operate at a comparable scanning time as today's C-arm based system, but with a better imaging quality.

\section{References}

[1] Wu, T., et al. (2003) Tomographic Mammography Using a Limited Number of Low-Dose Cone-Beam Projection Images. Medical Physics, 30, 365-380. 
https://doi.org/10.1118/1.1543934

[2] Chen, Y., Lo, J.Y. and Dobbins, J.T. (2005) Impulse Response Analysis for Several Digital Tomosynthesis Mammography Reconstruction Algorithms. Proceedings of SPIE, 5745, 541-549. https://doi.org/10.1117/12.595684

[3] Bissonnette, M., et al. (2005) Digital Breast Tomosynthesis Using an Amorphous Selenium Flat Panel Detector. Proceedings of SPIE, 5745, 529-540. https://doi.org/10.1117/12.601622

[4] Kopans, D.B. (1997) Breast Imaging. 2th Edition, Lippincott Williams and Wilkins, New York.

[5] Schardt, P., et al. (2004) New X-Ray Tube Performance in Computed Tomography by Introducing the Rotating Envelope Tube Technology. Medical Physics, 31, 2699-2706. https://doi.org/10.1118/1.1783552

[6] Zhang, J., et al. (2005) A Stationary Scanning X-Ray Source Based on Carbon Nanotube Field Emitters. Applied Physics Letters, 86, Article ID: 184104. https://doi.org/10.1063/1.1923750

[7] Zhou, O., Lu, J.P. and Qiu, Q. (2005) Large-Area Individually Addressable Multi-Beam X-Ray System and Method of Forming Same. US Patent No. 6876724.

[8] Good, W., et al. (2008) Digital Breast Tomosynthesis: A Pilot Observer Study. American Journal of Roentgenology, 190, 865-869.

[9] Ren, B., et al. (2005) Design and Performance of the Prototype Full Field Breast Tomosynthesis System with Selenium Based Flat Panel Detector. Proceedings of SPIE, 5745, 550-561. https://doi.org/10.1117/12.595833

[10] Qian, X., et al. (2009) Design and Characterization of a Spatially Distributed Multibeam Field Emission X-Ray Source for Stationary Digital Breast Tomosynthesis. Medical Physics, 36, 11. https://doi.org/10.1118/1.3213520

[11] Qian, X., Lu, J.P. and Zhou, O. (2012) High Resolution Stationary Digital Breast Tomosynthesis Using Distributed Carbon Nanotube X-Ray Source Array. Medical Physics, 39, 2090-2099. https://doi.org/10.1118/1.3694667

[12] Stevens, G.M., et al. (2003) Circular Tomosynthesis: Potential in Imaging of Breast and Upper Cervical Spine-Preliminary Phantom and in vitro Study. Radiology, 228, 569-575. https://doi.org/10.1148/radiol.2282020295

[13] Suryanarayanan, S., et al. (2000) Comparison of Tomosynthesis Methods Used with Digital Mammography. Academic Radiology, 7, 1085-1097. https://doi.org/10.1016/S1076-6332(00)80061-6

[14] Baptist, R. (2001) X-Ray Tube Comprising an Electron Source with Microtips and Magnetic Guiding Means. US Patent No. US6259765B1.

[15] Whitlock, R.R., et al. (2001) Transmission Cathodes for X-Ray Production. US Patent No. US6333968B1.

[16] Mckinley, R.L., et al. (2004) Simulation Study of a Quasi-Monochromatic Beam for X-Ray Computed Mammotomography. Medical Physics, 31, 800. https://doi.org/10.1118/1.1668371

[17] Crotty, D.J., McKinley, R.L. and Tornai, M.P. (2007) Experimental Spectral Measurements of Heavy K-Edge Filtered Beams for X-Ray Computed Mammotomography. Physics in Medicine \& Biology, 52, 603. https://doi.org/10.1088/0031-9155/52/3/005 